\title{
Solvent-Free MgO-Functionalized Mesoporous Catalysts for Jatropha Oil Transesterification
}

\author{
Anamagreth Andrew, Jamidu Katima, ${ }^{1}$ \\ Keat Teong Lee, ${ }^{2}$ and James Epiphan Gabriel Mdoe $^{3}$ \\ ${ }^{1}$ Chemical and Mining Engineering Department, College of Engineering and Technology, University of Dar es Salaam, \\ P.O. Box 35131, Dar es Salaam, Tanzania \\ ${ }^{2}$ School of Chemical Engineering, Universiti Sains Malaysia, Engineering Campus, Seri Ampangan, 14300 Nibong Tebal, \\ Pulau Pinang, Malaysia \\ ${ }^{3}$ Chemistry Department, College of Natural and Applied Sciences, University of Dar es Salaam, P.O. Box 35065, \\ Dar es Salaam, Tanzania \\ Correspondence should be addressed to Anamagreth Andrew; annamaggy86@yahoo.com
}

Received 9 January 2015; Accepted 20 May 2015

Academic Editor: E. Goldys

Copyright (C) 2015 Anamagreth Andrew et al. This is an open access article distributed under the Creative Commons Attribution License, which permits unrestricted use, distribution, and reproduction in any medium, provided the original work is properly cited.

A convenient solvent-free technique was employed in the functionalization of Micelle-Templated Silica using Cashew Nut Shell Liquid (MTS-CNSL) as a template and magnesium nitrate as a precursor salt. Magnesium oxide species was highly dispersed in MTS-CNSL by manually grinding the precursor salt and the as-synthesized mesoporous silica followed by calcination. The resultant modified mesoporous silicas MgO/MTS-CNSL were characterized by X-ray diffraction (XRD), Fourier transform infrared spectroscopy (FT-IR, $\mathrm{N}_{2}$ adsorption/desorption), and scanning electron microscopy/energy dispersive X-ray (SEM/EDX). $\mathrm{MgO} / \mathrm{MTS}$-CNSL (30) having small specific surface area of $16.7 \mathrm{~m}^{2} / \mathrm{g}$ and larger pore volume of $0.02 \mathrm{~cm}^{3} / \mathrm{g}$, presented higher activity of $81.45 \%$ for jatropha oil under optimized conditions $\left(200^{\circ} \mathrm{C}, 4 \mathrm{~h}, 36: 1\right.$ methanol : oil ratio, $500 \mathrm{rpm}$, and $6 \%$ wt of catalyst). This method of catalyst development has an advantage of being highly energy- and time-efficient.

\section{Introduction}

The intensification of porous materials with large specific surface areas is presently an area of extensive research, mainly with regard to potential applications in areas such as catalysis. In the early 1990s Koruda group and Mobil Company discovered mesoporous materials which they classified as microporous $(<2 \mathrm{~nm})$, mesoporous $(2-50 \mathrm{~nm})$, and macroporous $(>50 \mathrm{~nm}$ ) materials [1]. This class of materials is characterized by well-defined pore radius distributions, very large specific surface areas, and ordered pore systems [2]. In this class of silicas, the most distinguished representatives include the silica solids MCM-50 (with a laminar structure, space group p2), MCM-48 (with a cubic arrangement of the mesopores, space group Ia3d), and MCM-41 (with hexagonal arrangement of the mesopores, space group p $6 \mathrm{~mm}$ ).
Nonetheless, these mesoporous silicas experience an intrinsic lack of active sites necessary for catalysis; therefore their functionalization has become paramount [3].

To facilitate their application in industry, various metals, metal oxides, and organic and inorganic groups' functionalities have been incorporated by direct synthesis (one-pot method) or postsynthesis. The most commonly employed method is impregnation with solutions of simple thermally unstable precursor salts (e.g., nitrates, chlorides, and acetates) $[4,5]$. To facilitate molecular dispersion of oxide species, normally organic metal precursors are used to react with acidic silanols group as bases and then grafted on the surface of ordered mesoporous materials. Nevertheless, this grafting method usually achieves lower loading than the former one.

However, most of these processes require using a solution, which not only causes waste but also requires extra energy 

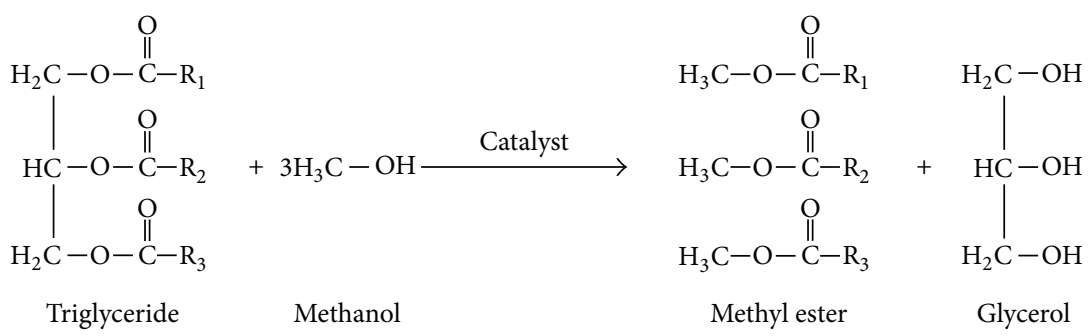

FIGURE 1: Chemistry of transesterification reaction.

to remove the solvent. During impregnation-calcinations processes, the surface of support undergoes the soakagedrying procedure, which changes not only the distribution or dispersion the oxide species, but also the surface state of the host. Moreover, competitive adsorption of solvent molecules will disturb the guest-host interaction, which may hinder the dispersion and anchoring of the active component species [6].

Loading via the gas phase by a volatile precursor and subsequent calcinations and the solid-state exchange are the other two less popular methods [7, 8]. Few solid-state studies are devoted to the supported mesoporous materials though solid-state exchange has been extensively studied in the synthesis of modified zeolites [9]. In this study, in order to inquire about solvent-free modification of mesoporous materials, we tried to disperse metal oxide species on the Cashew Nut Shell Liquid-Templated Mesoporous Silica (MTS-CNSL), by finely grinding the metal precursor salt, and support the following subsequent calcinations. This method utilizes the reactivity of the rich surface silanols groups of mesoporous support, using nitrate to suppress the tendency of metal to agglomerate into metal oxide domains and providing an effective means to disperse oxide species finely [6]. Being solvent-free process, this method has an advantage of being energy- and timeefficient. The developed catalyst will then be used in the transesterification of jatropha oil (Figure 1).

\section{Materials and Methods}

2.1. Catalyst Preparation. Jatropha oil (Diligent (T) Ltd., Tanzania), cashew nut shells (Uvungi, Tanzania), hexane, aqueous ammonia (33\%), absolute ethanol (95\%), absolute methanol (95\%), tetraethyl-orthosilicate (TEOS), sodium hydroxide, and magnesium nitrate purchased from SigmaAldrich (Malaysia) were used as received. All chemicals were of analytical grade and were used without purification. Micelle-Templated Silica (MTS) was prepared using Cashew Nut Shell Liquid (CNSL) as a surfactant and tetraethyl orthosilicate (TEOS) as the silica source. Typically, $5 \mathrm{~g}$ of CNSL was added to an aqueous solution of ethanol $(104 \mathrm{~mL}$ of absolute ethanol and $106 \mathrm{~mL}$ of distilled water) at room temperature. TEOS $36.67 \mathrm{~g}$ was added to the mixture and the mixture was adjusted to $\mathrm{pH}$ of 11 by addition of ammonia solution dropwise. The thick paste obtained was filtered and washed with $100 \mathrm{~mL}$ hot methanol and $100 \mathrm{~mL}$ distilled water after $24 \mathrm{~h}$ of continuous stirring. Afterwards the resulting white solid was dried for $24 \mathrm{~h}$ at $100^{\circ} \mathrm{C}$, without calcination. Precursor magnesium nitrate was manually ground with the as-prepared MTS-CNSL at room temperature. In general, the resulting homogeneous powder was calcined at $500^{\circ} \mathrm{C}$ for $5 \mathrm{~h}$ in order to remove template. The resultant $\mathrm{MgO} / \mathrm{MTS}$ CNSL samples were denoted as MgO/MTS-CNSL $(x)$, where $x$ represents the mass percentage of $\mathrm{MgO}$ in the composite.

2.2. Catalyst Characterization. The pore volume, specific surface area, and pore size distribution properties of the catalyst were measured by nitrogen adsorption/desorption isotherms using an ASAP 2020 Micromeritics instrument at $77 \mathrm{~K}$ by Brunauer-Emmett-Teller (BET) method. Before each measurement the samples were first degassed at $300^{\circ} \mathrm{C}$ for $2 \mathrm{~h}$ and thereafter kept at liquid nitrogen temperature to adsorb nitrogen. Scanning electron microscopy/energy dispersive X-ray (SEM/EDX) using QUANTA FEG 250 operated at $5.0 \mathrm{kV}$ was carried out to study the catalysts morphology. The samples were evenly distributed on a black doubled carbon tape attached to the aluminium stub and vacuumed for about $10 \mathrm{~min}$ for analyses. Energy dispersive X-ray (EDX) spectrum was used to calculate the ratio between cations for each sample by using ISIS Oxford Microanalysis facility of the system. XRD Philip PW 1820 diffractometer using Cu K $\alpha$ radiation $(\lambda=1.5418 \AA)$ was used to characterize crystalline structure of the catalysts. The X-ray tube was operated at $40 \mathrm{kV}$ and $120 \mathrm{~mA}$. Fourier transform infrared (FT-IR) analysis was carried out on the catalysts and the spectra for FT-IR analysis were recorded from 4000 to $1000 \mathrm{~cm}^{-1}$. The samples and analytical grade Potassium bromide $\mathrm{KBr}$ were dried at $80^{\circ} \mathrm{C}$ overnight prior to the FT-IR analysis.

2.3. Catalytic Test. $30 \mathrm{~g}$ of jatropha oil, $39.68 \mathrm{~g}$ of methanol, and $6 \%$ wt of MgO/MTS-CNSL $(x)$ catalyst concentrations were mixed in autoclave batch reactor with a magnetic stirrer ( $x$ stands for 30,50 , and $70 \% \mathrm{MgO}$ loading). The mixture was heated up to $200^{\circ} \mathrm{C}$ with stirring at $500 \mathrm{rpm}$ for $4 \mathrm{~h}$. The product and byproduct were left to separate overnight and upper layer which is the methyl ester was taken for analysis by a gas chromatography (PerkinElmer Clarus 500).

2.4. Analysis. The content of FAME in the resulting products was analyzed using gas chromatography (GC) equipped with flame ionization detector and Nukol column $(15 \mathrm{~m} \times$ $0.53 \mathrm{~mm} \times 0.5 \mathrm{~mm})$. Oven temperature was set at $110^{\circ} \mathrm{C}$ and temperature for injector and detector were set at $220^{\circ} \mathrm{C}$ and $250^{\circ} \mathrm{C}$, respectively. Helium was used as the carrier gas with flow rate of $10 \mathrm{~mL} / \mathrm{min}$. A quantity of $1 \mu \mathrm{L}$ from each sample was injected into the column. Methyl heptadecanoate was 


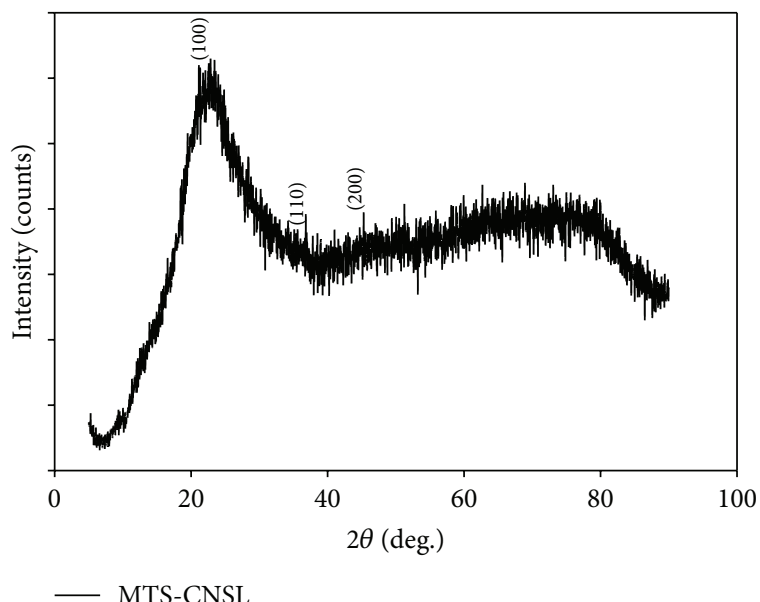

(a)

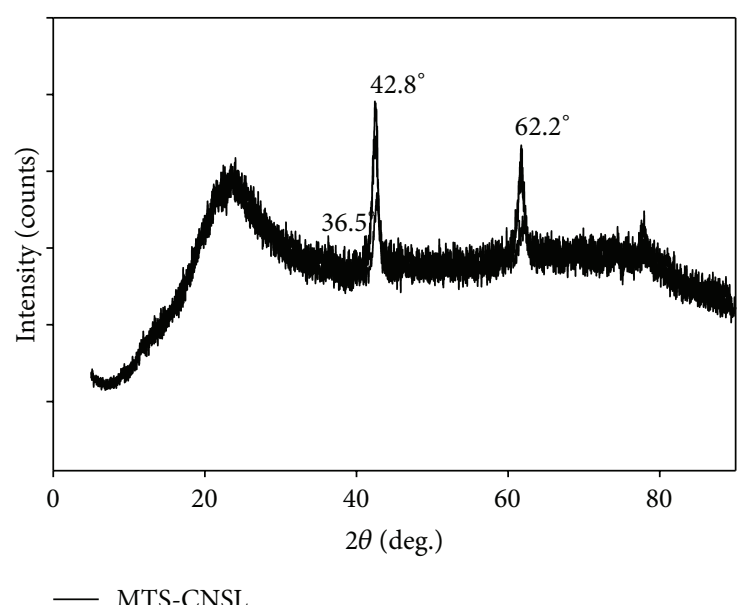

(b)

FIgure 2: XRD diffraction patterns of (a) MTS-CNSL and (b) MgO/MTS-CNSL samples.

used as internal standard for the calculation of the yield. The yield of the transesterification process was calculated as follows:

$$
\text { Yield }=\frac{\sum A-A_{\mathrm{IS}}}{A_{\mathrm{IS}}} \times \frac{C_{\mathrm{IS}} \times V_{\mathrm{IS}}}{m} \times 100 \%,
$$

where $\sum A$ is the total peak area from the methyl ester; $A_{\mathrm{IS}}$ is the internal standard (methyl heptadecanoate) peak area; $C_{\mathrm{IS}}$ is the concentration of the internal standard solution, in $\mathrm{mg} / \mathrm{mL} ; V_{\text {IS }}$ is the volume of the internal standard solution used, in $\mathrm{mL}$; and $m$, is the mass of the sample, in $\mathrm{mg}$.

\section{Results and Discussion}

3.1. Catalyst Characterization. The XRD pattern of calcined mesoporous silica shows an intense peak along with a broad shoulder (Figure 2(a)). This pattern is a characteristic of a mesophase with a pore system lacking long-range order which is a reminiscent of the disordered mesoporous silicas prepared by nonionic surfactant templating [10]. The variations of the peak intensity in the XRD patterns were recorded for the surface-modified samples and an intense diffraction peak for the samples as shown in Figure 2(b). Additional reflections of (2 $\left.\begin{array}{lll}2 & 0\end{array}\right),\left(\begin{array}{lll}1 & 1 & 0\end{array}\right)$, and $\left(\begin{array}{lll}1 & 0 & 0\end{array}\right)$ peaks which are usually observed in mesoporous materials were also observed in these samples. MTS-CNSL showed Bragg plane pattern $\left(\begin{array}{lll}1 & 0 & 0\end{array}\right),\left(\begin{array}{lll}1 & 1 & 0\end{array}\right)$, and $\left(\begin{array}{lll}2 & 0 & 0\end{array}\right)$ reflections, which are characteristic of a two-dimensional (2D) hexagonal (p6mm) structure [11]. This pointed out that the addition of a controlled amount of precursor salts does not apparently change the mesoscopic order of the MTS-CNSL host [12]. The presence of $\left(\begin{array}{lll}1 & 1 & 0\end{array}\right)$ reflection is known to be a phase transition from the cubic mesostructure to the tetragonal mesostructure after removal of silica template [13]. Broadening of ( $\left.\begin{array}{lll}2 & 0 & 0\end{array}\right)$ peak compared to MTS-CNSL shows to some extent loss of mesostructural orders in the $\mathrm{MgO} / \mathrm{MTS}$-CNSL samples due to disordered mesostructures.
However, the reflection $\left(\begin{array}{lll}1 & 0 & 0\end{array}\right)$ of the impregnated catalysts tends to shift to a higher angle, indicative of a lower d100 spacing with the introduction of magnesium ion [12]. Peaks $2 \theta$ at $42.8^{\circ}$ and $62.2^{\circ}$ correspond well with the $\left(\begin{array}{lll}2 & 0 & 0\end{array}\right)$ and (2 $\left.2 \begin{array}{ll}2 & 0\end{array}\right)$ reflections of $\mathrm{MgO}$ in periclase structure [14].

Figures 3 and 4 show representative SEM micrographs of calcined mesoporous silica and supported catalysts. It can be observed that the support and supported catalysts consist almost entirely of spherical particles, although some agglomeration is evident. The anchored materials are seen in the micrographs of supported catalysts as randomly distributed species. However, this effect is significant in the materials prepared using CNSL templates [15]. It is worth mentioning that the exterior surfaces of these spheres are very smooth. The large size of materials prepared by using CNSL templates may be due to a large phenolic head group in CNSL. For the $\mathrm{MgO} / \mathrm{MTS}-\mathrm{CNSL}$ (30), the silica-magnesium distribution was more heterogeneous, suggestive of the formation of silica-magnesia domains and separated silica [16] and this might be the reason contributing to the high conversion obtained during transesterification reaction of jatropha oil.

The SEM images clearly show that in general particle sizes and morphologies of mesoporous materials of $\mathrm{MgO}$ are very similar to those of templated silicas. In this present study, magnesium nitrate precursor was impregnated into the mesopores without using any solvent by simply heating a physical mixture of precursor salt and mesoporous silica. EDX analysis (Figure 5) of Mg/MTS-CNSL (30) is composed of $\mathrm{Si}, \mathrm{O}$, and $\mathrm{Mg}$ elements, which corroborate the presence of $\mathrm{MgO}$ in MTS-CNSL.

Fourier transform infrared spectroscopy (FT-IR) was used in the qualitative determination of functional groups on the supported catalysts. The broad band (Figure 6) around $3460 \mathrm{~cm}^{-1}$ could be ascribed to the adsorbed water and to the $\mathrm{H}$-bonded silanols $\mathrm{OH}$ groups [17]. The intensity of the band around $1650 \mathrm{~cm}^{-1}$ is predicted to be the bending vibration of water molecule physisorbed [18]. The major peaks of MTS-CNSL were observed in the FT-IR spectra of calcined 


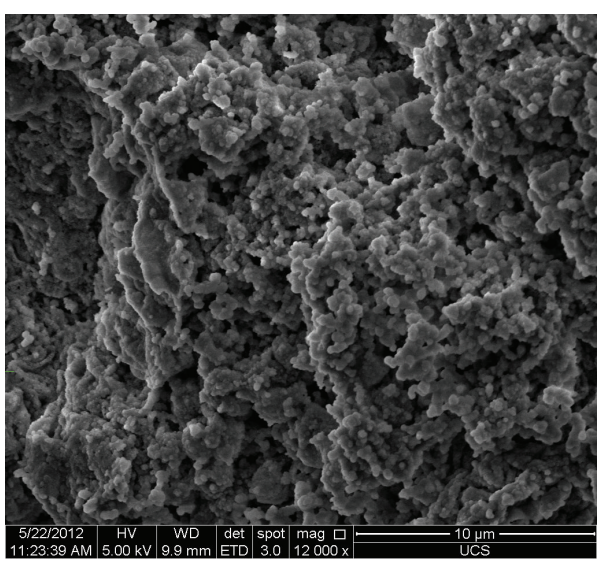

(a)

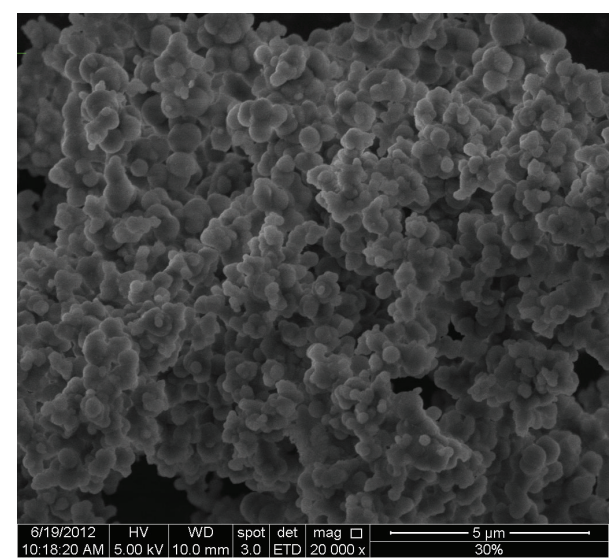

(b)

Figure 3: Scanning electron micrograph of (a) MTS-CNSL; (b) scanning electron micrograph of MgO/MTS-CNSL (30).

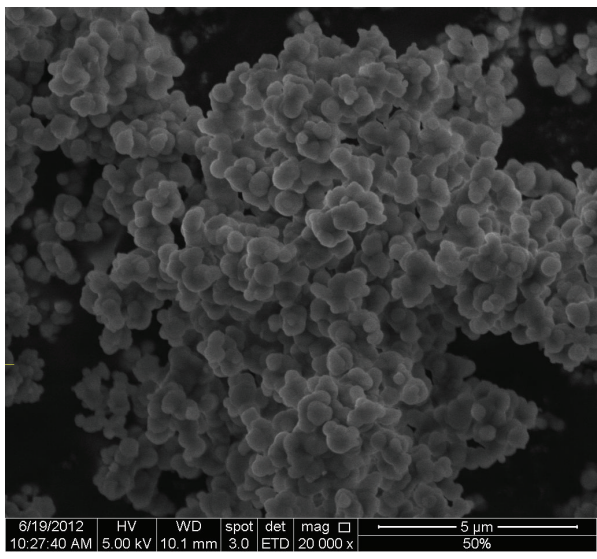

(a)

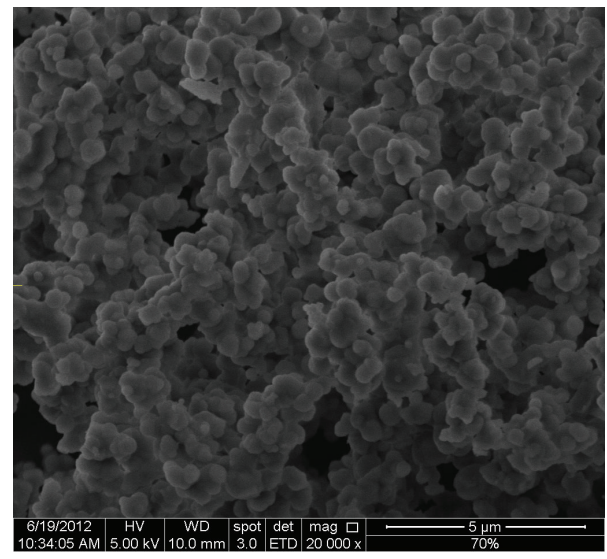

(b)

Figure 4: Scanning electron micrograph of (a) MTS-CNSL (50); (b) scanning electron micrograph of MgO/MTS-CNSL (70).

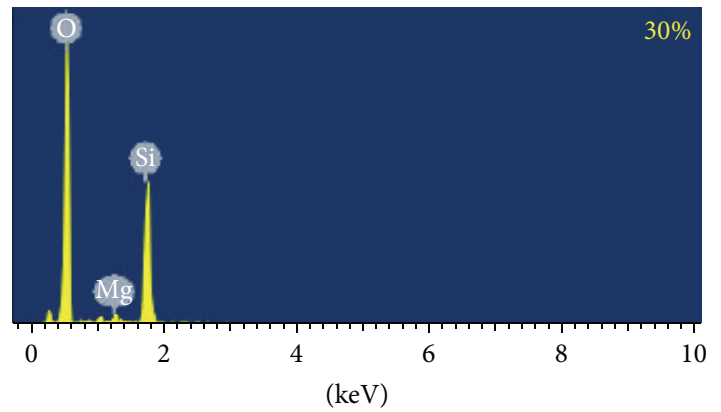

Full-scale 672 cts cursor: -0.265 ( 0 cts)

Figure 5: EDX of MgO/MTS-CNSL (30).

MTS-CNSL that consist of asymmetrical Si-O-Si stretching vibration in the range between 1200 and $1000 \mathrm{~cm}^{-1}$ which is $1100 \mathrm{~cm}^{-1}$, symmetrical $\mathrm{Si}-\mathrm{O}-\mathrm{Si}$ stretching vibration at $802 \mathrm{~cm}^{-1}$, Si-O-Si bending at $460 \mathrm{~cm}^{-1}$, and Si-OH stretching vibration at $970 \mathrm{~cm}^{-1}[19]$.
The intensity of the $960 \mathrm{~cm}^{-1}$ band was degenerated to the shoulder of the $1080 \mathrm{~cm}^{-1}$ band with the modification of MTS-CNSL with $\mathrm{MgO}$, which points out that the surface silanols groups around $960 \mathrm{~cm}^{-1}$ interrelated with the oxide species and contributed to forming the $\mathrm{Si}-\mathrm{O}-\mathrm{Mg}$ in the process of modification. Usually the band $960 \mathrm{~cm}^{-1}$ is taken as evidence for the incorporation of metal atoms in the silica framework of mesoporous material [20]. This agrees with the FT-IR results, whereby around $960 \mathrm{~cm}^{-1}$ no Si-OH bending bands are observed on the mesoporous catalysts spectrums. This supports the interaction between the magnesium oxide species and the host. Nitrate anions have a $\pi$ system electronic structure and act as bidentate ligands to form hydrogen bonds with the silanols easily. Hence nitrate ions favor formation of Si-O-Mg during heating.

The specific surface area, pore volume, and mean pore diameter were obtained from nitrogen gas adsorptiondesorption studies. Figure 7 shows the obtained nitrogen gas adsorption-desorption isotherms for MgO/MTS-CNSL (30). It can be seen that a type IV isotherm with capillary condensation step at high relative pressures is observed and a 


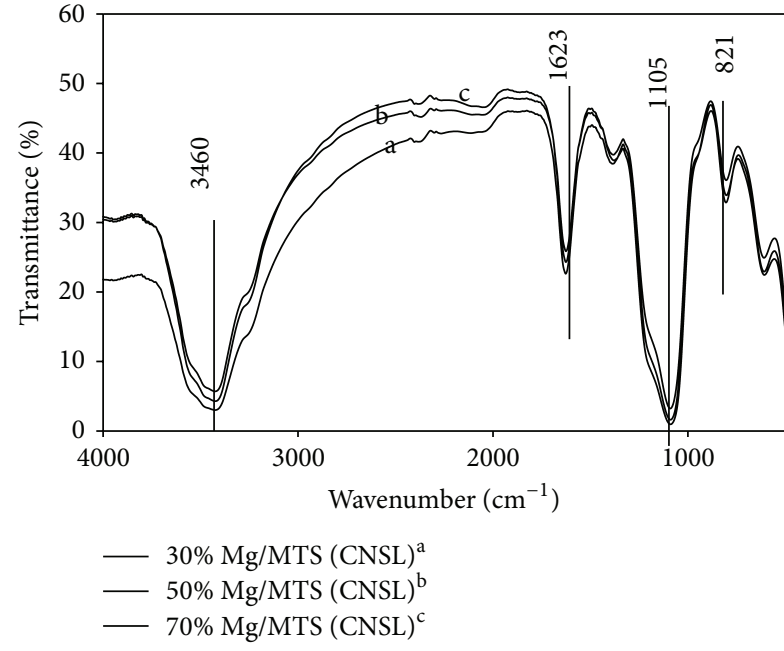

(a)

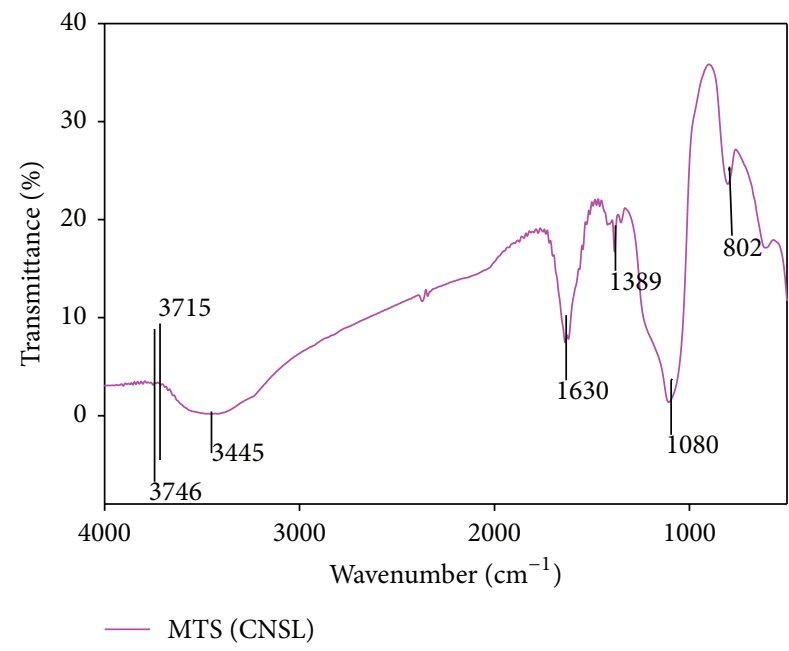

(b)

FIGURE 6: FT-IR transmission spectrum of (a) MgO/MTS-CNSL catalysts; (b) MTS-CNSL silica.

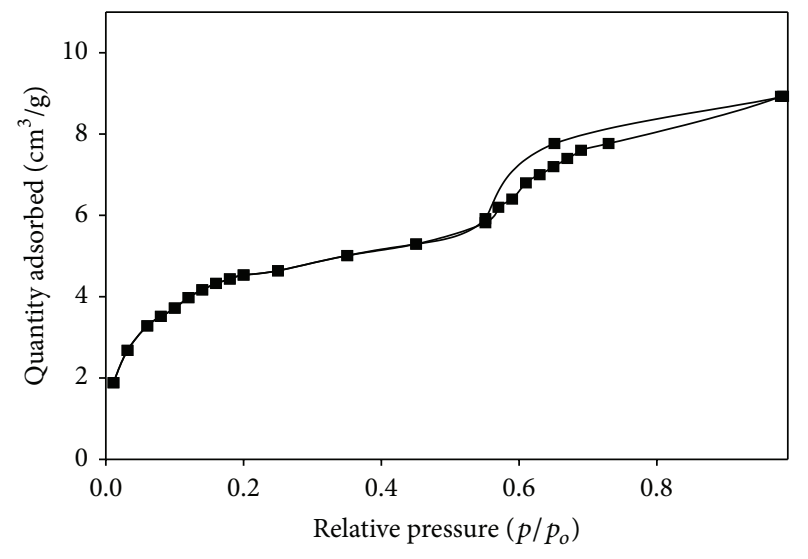

FIGURE 7: Nitrogen gas adsorption-desorption isotherms for $\mathrm{MgO} / \mathrm{MTS}-\mathrm{CNSL}$ (30).

TABLE 1: Characteristics of the developed mesoporous catalysts.

\begin{tabular}{lccc}
\hline Catalyst & $\begin{array}{c}\text { Specific } \\
\text { surface area } \\
(\mathrm{BET}) / \mathrm{m}^{2} \mathrm{~g}^{-1}\end{array}$ & $\begin{array}{c}\text { Volume of } \\
\text { pores }\left(\mathrm{cm}^{3} / \mathrm{g}\right)\end{array}$ & $\begin{array}{c}\text { Mean pore } \\
\text { diameter } \\
(\mathrm{nm})\end{array}$ \\
\hline MgO/MTS-CNSL (30) & 16.7 & 0.020 & 46.85 \\
MgO/MTS-CNSL (50) & 30 & 0.019 & 18.64 \\
MgO/MTS-CNSL (70) & 40 & 0.014 & 18.43 \\
MTS-CNSL & 13.26 & 0.010 & 30.17 \\
\hline
\end{tabular}

hysteresis loop near pressure 0.60 in the desorption branch indicates the presence of mesopores [21]. The measured results reveal that $\mathrm{MgO} / \mathrm{MTS}$-CNSL materials exhibit defined mesostructures.

From Table 1, MgO/MTS-CNSL (70) had larger surface area compared to other prepared catalysts. This might be due to availability of more magnesium ion which has larger ionic radii equal to $\mathrm{Si}^{2+}$ ion. MgO/MTS-CNSL (30) had smaller
TABLE 2: Transesterification of jatropha oil over different catalysts.

\begin{tabular}{lc}
\hline Catalyst & Yield of ME (\%) \\
\hline MgO/MTS-CNSL (30) & 81.45 \\
MgO/MTS-CNSL (50) & 78.57 \\
MgO/MTS-CNSL (70) & 77.66 \\
MTS-CNSL & 13.46 \\
No catalyst & 12.48 \\
\hline
\end{tabular}

surface area compared to other catalysts, but it had larger pore diameter, hence providing active surface where the reactants can react. It thus has the propensity to provide adequate porosity for the reacting molecules.

3.2. Transesterification Reaction. Transesterification reaction of jatropha oil was used to examine the catalytic activity of the supported catalysts loaded with $\mathrm{MgO}$ at different weight percentages (30,50, and 70\%). Reaction conditions were $200^{\circ} \mathrm{C}, 4 \mathrm{~h}$ reaction time with $6 \% \mathrm{wt}$ of catalyst, methanol to oil ratio and mixing rate being set at $36: 1$ and $500 \mathrm{rpm}$, respectively.

Figure 8 shows that MgO/MTS-CNSL (30) had higher catalytic activity compared to other catalysts, while the ester conversions of the reactions catalyzed by pure silica without loading showed almost similar conversion to that of the control experiment (Table 2), where no catalyst was used. It is obvious that pore radius, surface area, and pore volume cannot straightforwardly explain the activity of $\mathrm{MgO}$ with respect to the yield of methyl esters, though the interaction between pore diameter and surface area is vital in ensuring diffusion to the active sites. This explains that it may be possible that other structural factors may affect the activity of $\mathrm{MgO}$. It has been once reported that base sites over heterogeneous catalysts are the active centers for transesterification reaction. The number and basic strength of the active sites are one possibility that affects the $\mathrm{MgO}$ activity [22]. The basicity 


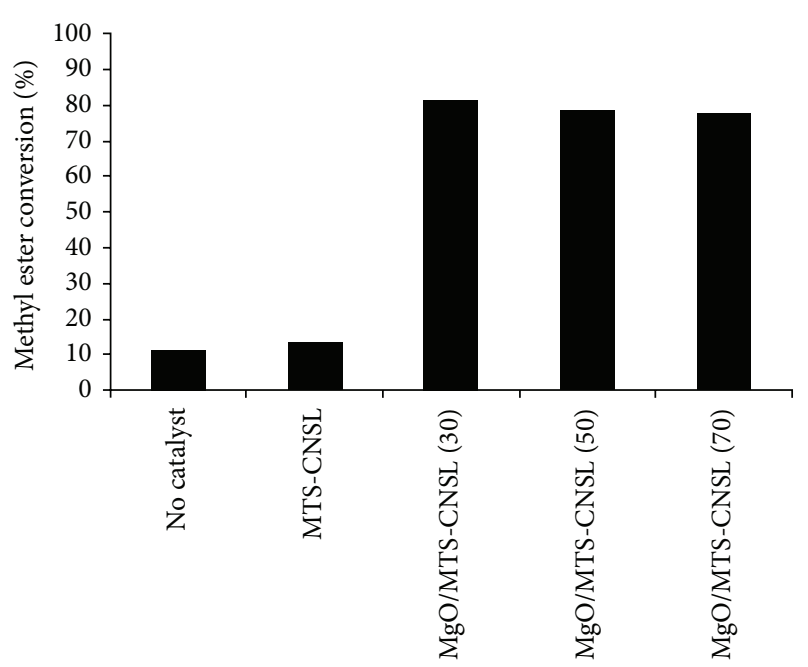

FIGURE 8: Methyl ester conversions of transesterification reactions.

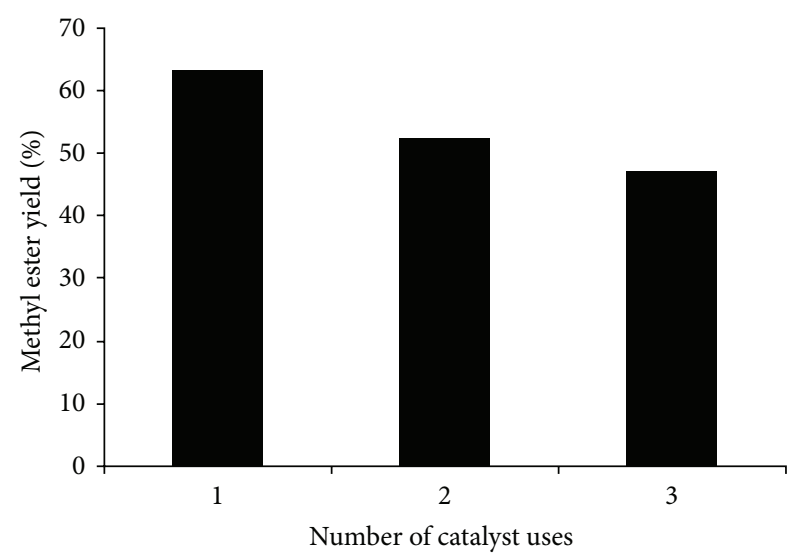

Figure 9: Catalyst reuses MgO/MTS-CNSL (30). Reaction conditions: $200^{\circ} \mathrm{C}$ for $4 \mathrm{~h}$, catalyst $(6.0 \% \mathrm{wt})$.

of the developed catalysts was tested using the method by [23]; MgO/MTS-CNSL (30) was found to have higher basicity of $2379 \mu \mathrm{mol} / \mathrm{g}$ compared to other catalysts and had a positive effect on transesterification reaction. Catalytic activities of MgO/MTS-CNSL (50) and MgO/MTS-CNSL (70) were shown to decrease, and this may be due to the formation of new weaker basic sites $[23,24]$. Though MgO/MTS-CNSL (30) had smaller surface area, its conversion was higher compared to other catalysts. It suggests that MgO/MTSCNSL (30) has pores wider than other developed catalysts and also has as small number of pores. This can be explained by the fact that higher specific surface area materials in general comprise a high proportion of very small pores and thus will lead to severe diffusion limitations in this complex threephase reaction [22]. The high pore volume of the MgO/MTSCNSL (30) favored the reaction by rendering the active sites more accessible to the bulky triglycerides molecules.

3.3. The Recycling of the Catalyst. One of the main disadvantages of homogeneous catalysts is that they cannot be reused. But heterogeneous catalysts can be recovered, regenerated, and reused. Reusability studies were carried out using the most active catalyst MgO/MTS-CNSL (30), whereby it was reused three times for the transesterification reaction. There is a possibility that catalyst was poisoned by reaction medium, for example, glycerol, oil, and methyl ester. Therefore, extra washing step and thermal treatment are necessary. Recovery of catalyst was done by filtering, washing with hexane and ethanol, dried at $80^{\circ} \mathrm{C}$ for $2 \mathrm{~h}$, and calcined at $500^{\circ} \mathrm{C}$. As shown in Figure 9, catalytic activity of MgO/MTSCNSL (30) decreased from $81.45 \%$ to $77.66 \%$. This might be due to successive calcinations which caused deactivation of the catalyst. Leaching affects significantly the industrial application of heterogeneous catalysts and reusability of catalyst. When the catalyst was tested for leaching, it was contacted with methanol at $60^{\circ} \mathrm{C}$ during $4 \mathrm{~h}$. Thereafter, the catalyst was filtered out and the activated methanol (with possible leached catalyst) was used in transesterification reaction with fresh reactants. The methyl ester yield (13.73\%) was almost similar to the one without catalyst (Table 2). The catalyst showed minor amount of catalyst leached into the methanol signifying that the developed catalysts were factual heterogeneous catalysts.

\section{Conclusion}

MgO/MTS-CNSL (30) was an efficient catalyst for the transesterification of jatropha oil with methanol exhibiting highest activity with methyl ester yield of $81.45 \%$ among the tested catalysts. The catalyst had larger pore diameter creating active sites more accessible to the bulky triglycerides molecules. Leaching of the catalyst was considered to be negligible as the reaction produced lower methyl ester yield of $13.73 \%$ almost similar to the reaction without catalyst. Reusability of catalyst was potential though the yield decreases to the third run due to hydrothermal treatment of the catalyst. The new catalysts prepared showed high activity for the synthesis of jatropha oil because of the presence of basic concerted active sites. Additionally, since the process is solvent-free, it has an advantage of being energy and time-saving ever since the mesopores can only be filled once with precursor. Furthermore, the current method can shorten the time-consuming replication process that needs the solvent filtering and drying step. Moreover, competitive adsorption of solvent molecules will disturb the guest-host interaction, which may hinder the dispersion and anchoring of the active component species.

\section{Conflict of Interests}

The authors declare that there is no conflict of interests regarding the publication of this paper.

\section{Acknowledgments}

The authors would like to thank the Swedish International Development Agency (Sida), Policy Innovation Systems for Clean Energy Security (PISCES) funded by the UK Department for International Development, the University of Dar es Salaam, and Universiti Sains Malaysia, School of Chemical Engineering, for equipment and financial support. 


\section{References}

[1] T. Yanagisawa, T. Shimizu, K. Kuroda, and C. Kato, “The preparation of alkyltrimethylammonium-kanemite complexes and their conversion to microporous materials," Bulletin of the Chemical Society of Japan, vol. 63, no. 4, pp. 988-992, 1990.

[2] M. Fröba, F. Hoffmann, M. Cornelius, and J. Morell, "Silicabased mesoporous organic-inorganic hybrid materials," Angewandte Chemie International Edition, vol. 45 , no. 20, pp. 32163251, 2006.

[3] A. Taguchi and F. Schüth, "Ordered mesoporous materials in catalysis," Microporous and Mesoporous Materials, vol. 77, no. 1, pp. 1-45, 2005.

[4] F. Schüth, A. Wingen, and J. Sauer, "Oxide loaded ordered mesoporous oxides for catalytic applications," Microporous and Mesoporous Materials, vol. 44-45, no. 1, pp. 465-476, 2001.

[5] J. Sauer, F. Marlow, B. Spliethoff, and F. Schüth, "Rare earth oxide coating of the walls of SBA-15," Chemistry of Materials, vol. 14, no. 1, pp. 217-224, 2002.

[6] Y. M. Wang, Z. Ying Wu, and J. H. Zhu, "Surface functionalization of SBA-15 by the solvent-free method," Journal of Solid State Chemistry, vol. 177, no. 10, pp. 3815-3823, 2004.

[7] S. T. Wong, H. P. Lin, and C. Y. Mou, "In-situ study of MCM-41supported iron oxide catalysts by XANES and EXAFS," Applied Catalalysis A, vol. 198, no. 1, pp. 103-115, 2000.

[8] P. van der Voort, M. Mathieu, E. F. Vansant, S. N. R. Rao, and M. G. White, "Molecular dispersions of metal complexes within microporous solids: an alternative way to prepare supported $\mathrm{MO}_{x}$ catalysts," Journal of Porous Materials, vol. 5, no. 3-4, pp. 305-316, 1998.

[9] J. Thoret, P. P. Man, E. Duprey, and J. Fraissard, "Interaction and solid-state reaction between manganese chloride tetrahydrate and Y zeolites ( $\mathrm{NaY}$ or LaNaY)," Journal of the Chemical Society-Faraday Transactions, vol. 94, no. 18, pp. 2867-2870, 1998.

[10] S. A. Bagshaw, E. Prouzet, and T. J. Pinnavaia, "Templating of mesoporous molecular sieves by nonionic polyethylene oxide surfactants," Science, vol. 269, no. 5228, pp. 1242-1244, 1995.

[11] H.-P. Lin and C.-Y. Mou, "Tubules-within-a-tubule hierarchical order of mesoporous molecular sieves in MCM-41," Science, vol. 273, no. 5276, pp. 765-768, 1996.

[12] E. Li and V. Rudolph, "Transesterification of vegetable oil to biodiesel over MgO-functionalized mesoporous catalysts," Energy \& Fuels, vol. 22, no. 1, pp. 145-149, 2008.

[13] L. A. Solovyov, V. I. Zaikovskii, A. N. Shmakov, O. V. Belousov, and R. Ryoo, "Framework characterization of mesostructured carbon CMK-1 by X-ray powder diffraction and electron microscopy," Journal of Physical Chemistry B, vol. 106, no. 47, pp. 12198-12202, 2002.

[14] B. Eckhardt, E. Ortel, J. Polte et al., "Micelle-templated mesoporous films of magnesium carbonate and magnesium oxide," Advanced Materials, vol. 24, no. 23, pp. 3115-3119, 2012.

[15] F. B. Hamad, E. B. Mubofu, and Y. M. M. Makame, "Wet oxidation of maleic acid by copper (II) Schiff base catalysts prepared using cashew nut shell liquid templates," Catalysis Science and Technology, vol. 1, no. 3, pp. 444-452, 2011.

[16] R. Brambilla, C. Radtke, J. H. Z. dos Santos, and M. S. L. Miranda, "Silica-magnesia mixed oxides prepared by a modified Stöber route: Structural and textural aspects," Powder Technology, vol. 198, no. 3, pp. 337-346, 2010.

[17] J. M. Berquier, L. Teyssedre, and C. Jacquiod, "Synthesis of transparent mesoporous and mesostructured thin silica films,"
Journal of Sol-Gel Science and Technology, vol. 13, no. 1-3, pp. 739-742, 1998.

[18] B. H. Hameed, L. F. Lai, and L. H. Chin, "Production of biodiesel from palm oil (Elaeis guineensis) using heterogeneous catalyst: an optimized process," Fuel Processing Technology, vol. 90, no. 4, pp. 606-610, 2009.

[19] R. Takahashi, S. Sato, T. Sodesawa, M. Kawakita, and K. Ogura, "High surface-area silica with controlled pore size prepared from nanocomposite of silica and citric acid," Journal of Physical Chemistry B, vol. 104, no. 51, pp. 12184-12191, 2000.

[20] T. Jiang, D. Wu, J. Song et al., "Synthesis and characterization of mesoporous ZrMCM-48 molecular sieves with good thermal and hydrothermal stability," Powder Technology, vol. 207, no. 13, pp. 422-427, 2011.

[21] D. S. Yuan, J. Zeng, J. Chen, and Y. Liu, "Highly ordered mesoporous carbon synthesized via in situ template for supercapacitors," International Journal of Electrochemical Science, vol. 4, no. 4, pp. 562-570, 2009.

[22] C. Xu, D. I. Enache, R. Lloyd, D. W. Knight, J. K. Bartley, and G. J. Hutchings, "Mgo catalysed triglyceride transesterification for biodiesel synthesis," Catalysis Letters, vol. 138, no. 1-2, pp. 1-7, 2010.

[23] T. Alemdaroglu, "Determination methods for the acidity of solid surfaces," Communications Faculty of Sciences University of Ankara B, vol. 47, pp. 27-35, 2001.

[24] K. G. Georgogianni, A. K. Katsoulidis, P. J. Pomonis, G. Manos, and M. G. Kontominas, "Transesterification of rapeseed oil for the production of biodiesel using homogeneous and heterogeneous catalysis," Fuel Processing Technology, vol. 90, no. 7-8, pp. 1016-1022, 2009. 

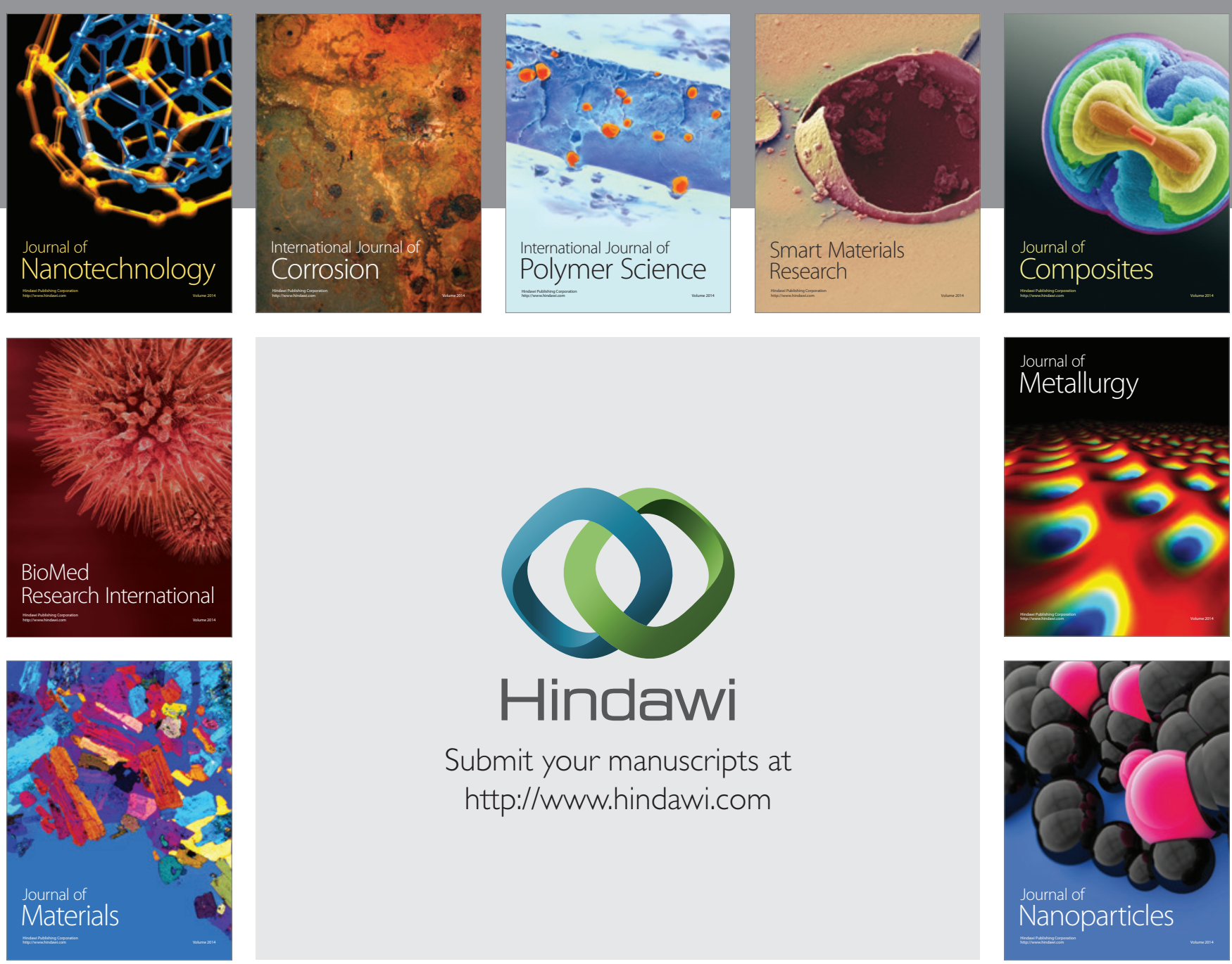

Submit your manuscripts at http://www.hindawi.com
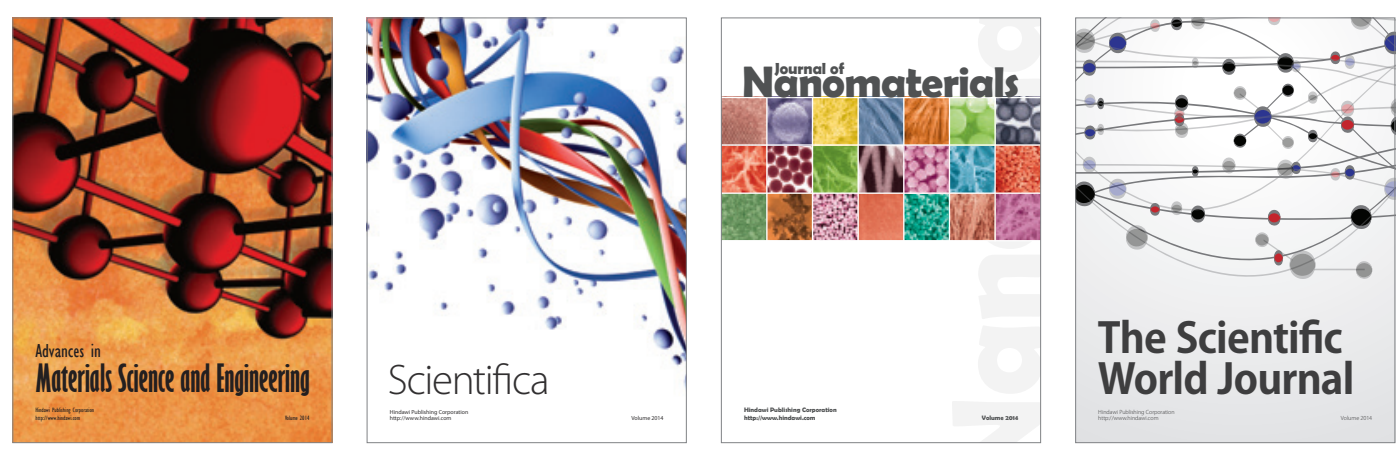

\section{The Scientific World Journal}
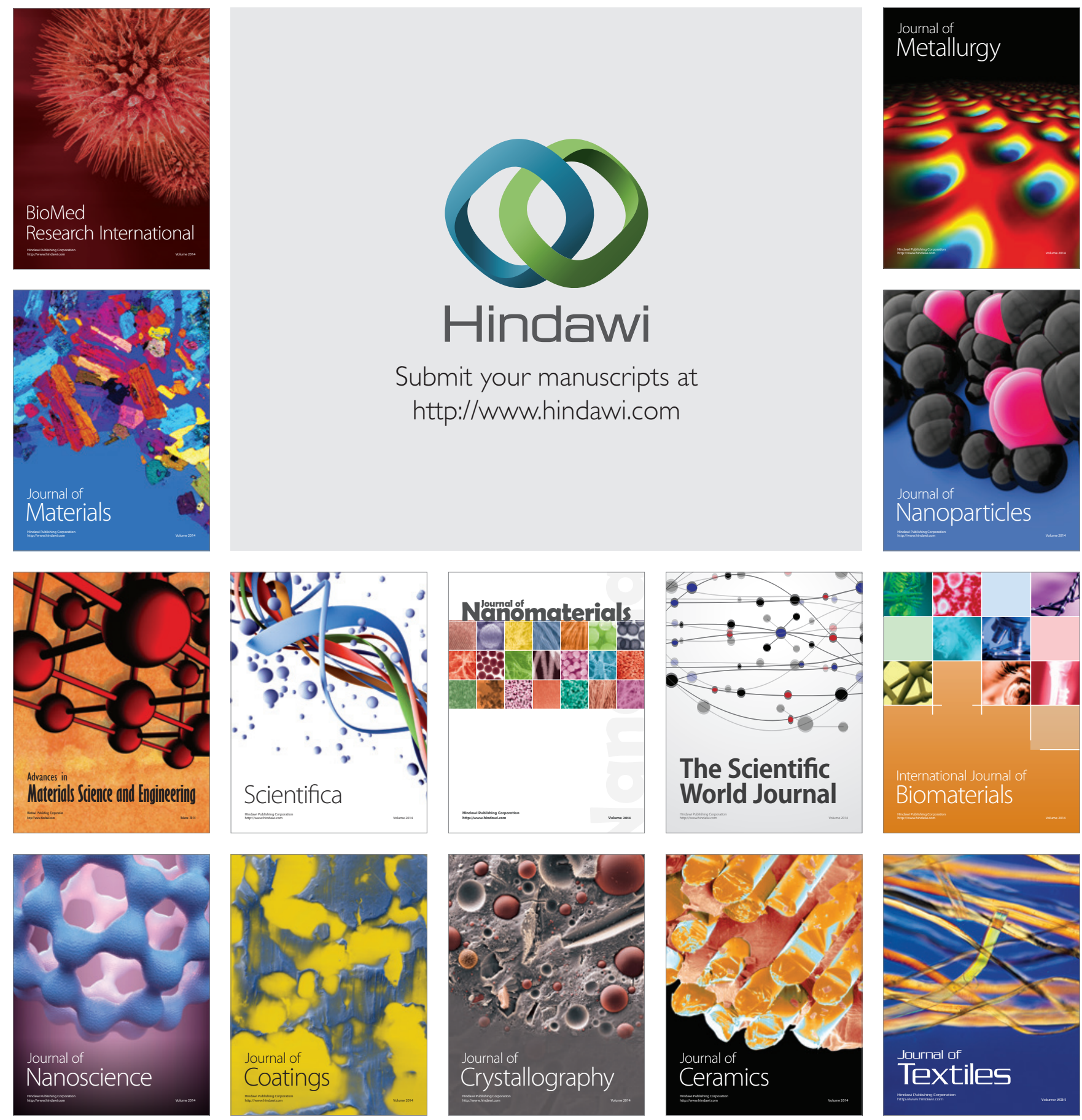